Pacific Journal of Mathematics

HOMOMORPHISMS OF MATRIX RINGS INTO MATRIX RINGS 


\title{
HOMOMORPHISMS OF MATRIX RINGS INTO MATRIX RINGS
}

\author{
Amos Kovacs
}

\begin{abstract}
Let $V_{n}\left(R_{n}\right)$ be the universal ring with respect to embeddings of the matrix ring $R_{n}$ into $n \times n$ matrix rings over commutative rings. A construction and a representation is given for this ring. As a main tool in the construction, it is proved that every $R$ homomorphism of $R_{n}, R$ a commutative ring, is the restriction of an inner automorphism of $U_{n}$, for some $U \supseteqq R$. Using this, a necessary and sufficient condition for $n^{2}$ matrices in $R_{n}$ to be matrix units is given.
\end{abstract}

1. Introduction and notations. All rings to be considered in this paper, except those denoted specifically as matrix rings, will be commutative rings with unit. All homomorphisms are unitary. The unit of a subring coincides with the unit of its over-ring.

Denote by $R_{n}$ the ring of $n \times n$ matrices over a ring $R$. Let $\eta: R \rightarrow S$ be a ring homomorphism then $\eta$ induces a homomorphism $\eta_{n}: R_{n} \rightarrow S_{n}$ given by: $\eta_{n}\left(r_{i j}\right)=\left(\eta\left(r_{i j}\right)\right)$. If $A \in R_{n},(A)_{i j}$ will denote the $(i, j)$ th entry of $A$. The identity element and the standard matrix units of all matrix rings will be denoted by $I$ and $\left\{E_{i j}\right\}$ respectively.

Let $A$ be an $R$ algebra. It was proved by Amitsur ([1], Theorem 2) that there exists a commutative $R$ algebra $V_{m}^{R}(A)$, and a map $\rho: A \rightarrow\left(V_{m}^{R}(A)\right)_{m}$ which is universal for homomorphisms of $A$ into $m \times m$ matrix rings over commutative rings, i.e.;

(1) For every $\tau: A \rightarrow H_{m}$, with $H$ a commutative $R$ algebra, there exists a homomorphism $\eta: V_{m}^{R}(A) \rightarrow H$ such that the following diagram is commutative;

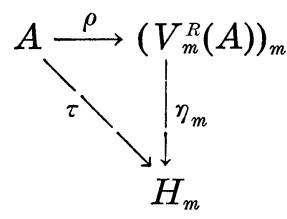

(2) $V_{m}^{R}(A)$ is generated over $R$ by the entries $\left\{[\rho(a)]_{i j} \mid a \in A\right\}$. Properties (1) and (2) determine $V_{m}^{R}(A)$ up to isomorphism and $\rho$ up to a multiple by an isomorphism of $V_{m}^{R}(A)$.

In this paper we will give an explicit construction for the ring $V_{m}^{R}\left(R_{n}\right)$. The case $n=m$ will be treated separately. We start with investigating the nature of $R$-homomorphisms of $R_{n}$ into itself. 


\section{On the automorphisms of matrix rings.}

Lemma 1. Let $C$ be a subdirectly irreducible commutative ring. $C$ can be embedded in a local ring $Q$ which is the complete ring of quotients of $C$. For noetherian $C$ we may take $C=Q$.

Proof. Let $\mathfrak{M}$ be the set zero divisors in $C$, then, by [6] $\mathfrak{M}$ is a maximal ideal in $C$. Let $Q$ be the local ring $C_{\mathrm{m}}$ and let $f$ be the canonical homomorphism $f: C \rightarrow C_{\mathrm{m}}$.

The elements of $C-\mathfrak{M}$ are not zero divisors in $C$, hence $f$ is an injection. Furthermore, the elements of $C-\mathfrak{M}$ are exactly the regular elements of $C$, and so $Q$ is the complete ring of quotients of $C$.

Now, if $C$ is noetherian we have, by [3], $\mathfrak{M}=\mathfrak{N}(C)$-the nil radical of $C$, and therefore, $J(C) \subseteq \mathfrak{M}=\mathfrak{R}(C) \leqq J(C)$ where $J(C)$ denotes the Jacobson radical of $C$. Hence $\mathfrak{M}=J(C)$ and being maximal it is the unique maximal ideal of $C$. Consequently $C$ is local and $C=C_{\mathrm{m}}=Q$.

Theorem 2. Let $C$ and $Q$ be as in the lemma; $\left\{E_{i j} \mid 1 \leqq i, j \leqq n\right\}$ be the set of the standard matrix units in $C_{n}$, and $\left\{F_{i j} \mid i \leqq i, j \leqq n\right\}$ another set of matrix units in $C_{n}$, then there exists a matrix $A \in C_{n}$, invertible in $Q_{n}$ such that:

$$
1 \leqq i, j \leqq n \quad E_{i j} A=A F_{i j} .
$$

If $C$ is noetherian $A$ is invertible in $C_{n}$.

Proof. By definition $\sum_{\nu=1}^{n} F_{\nu \nu}=I$ hence $\sum_{\nu=1}^{n}\left(F_{\nu \nu}\right)_{11}=\left(\sum_{\nu=1}^{n} F_{\nu \nu}\right)_{11}=1$. Now $C$ is subdirectly irreducible, so that the zero divisors in $C$ form an ideal. Consequently not all of the $\left(F_{\nu \nu}\right)_{11}$ are zero divisors and we have some $1 \leqq \nu \leqq n$ such that $\left(F_{\nu \nu}\right)_{11}$ is regular. Without loss of generality we may assume that $\alpha=\left(F_{11}\right)_{11}$ is regular. Put now

$$
A=\sum_{\nu=1}^{n} E_{\nu 1} F_{1 \nu} \quad B^{\prime}=\sum_{\nu=1}^{n} F_{\nu 1} E_{1 \nu}
$$

than for all $i$ and $j$ we have $E_{i j} A=E_{i 1} F_{1 j}=A F_{i j}$ and also

$$
A B^{\prime}=\left(\sum_{\nu=1}^{n} E_{\nu 1} F_{1 \nu}\right)\left(\sum_{\mu=1}^{n} F_{\mu 1} E_{1 \mu}\right)=\sum_{\nu=1}^{n} E_{\nu 1} F_{11} E_{1 \nu}=\alpha I .
$$

Now $\alpha$ is regular in $C$ and hence invertible in $Q$, thus $B=\alpha^{-1} B^{\prime}$ is the inverse of $A$ in $Q_{n}$. If $C$ is noetherian then by Lemma $1 C=Q$ and $B=A^{-1} \in C_{n}$.

Note that in a local ring the noninvertible elements form an ideal so that the proof of Theorem 2 can be easily modified to give 
an elementary proof of the following well-known (e.g. [2]) theorem: "Let $C$ be a local ring then every $C$-homomorphism of $C_{n}$ is an inner automorphism".

Theorem 3. Let $C$ be a commutative ring with unit, $E_{i j}, F_{i j} \in$ $C_{n}$ as above, then there exists a commutative ring $U$ containing $C$ and an invertible matrix $A \in U_{n}$ such that $E_{i j} A=A F_{i j}$ for all $1 \leqq$ $i, j \leqq n$.

Proof. $C$ may be represented as a subdirect product of subdirectly irreducible rings ([4], Theorem 1, p. 219). There exists therefore a set of subdirectly irreducible rings with unit, $\left\{C^{r} \mid \gamma \in \Gamma\right\}$ such that $C \leqq \prod_{r \in \Gamma} C^{\gamma} \leqq \Pi_{\gamma \in \Gamma} Q^{r}=U$ where $Q^{\gamma}$ is the complete ring of quotients of $C^{r}$. Hence $C_{n} \subseteq\left(\Pi C^{r}\right)_{n} \subseteq\left(\Pi Q^{r}\right)_{n}=U_{n}$. Let $\pi^{r}: \Pi Q^{r} \rightarrow$ $Q^{r}$ be the canonical projection. Put $E_{i j}^{\gamma}=\pi_{n}^{\gamma}\left(E_{i j}\right), F_{i j}^{r}=\pi_{n}^{r}\left(F_{i j}\right)$ then, by definition $E_{i j}^{\gamma}$ are the standard matrix units in $C^{\gamma}, F_{i j}^{\gamma}$ are another set of matrix units. By Theorem 2 it follows that there are invertible matrices $A^{r} \in Q_{n}^{r}$ such that $E_{i j}^{\gamma} A^{r}=A^{\gamma} F_{i j}^{\gamma}$. Let $A \in\left(\Pi Q^{r}\right)_{n}=$ $U_{n}$ be defined by $\left((A)_{\nu \mu}\right)_{(r)}=\left(A^{\gamma}\right)_{\nu \mu}$, namely for every $\gamma \in \Gamma \pi_{n}^{\gamma}(A)=$ $A^{r}$. Clearly $A$ is invertible in $U_{m}$, its inverse being given by $\left(\left(A^{-1}\right)_{\nu \mu}\right)_{(\gamma)}=\left(\left(A^{r}\right)^{-1}\right)_{\nu \mu}$. Clearly $A$ satisfies $E_{i j} A=A F_{i j}$ for all $i$ and $j$.

Corollary 4. (1) For a given ring $C$ there exists a ring $U \supseteqq C$ such that every C-homomorphism $\eta: C_{n} \rightarrow C_{n}$ can be extended to an inner automorphism of $U_{n}$.

(2) Given $\eta$, the ring $U$ of (1) may be chosen so that the inner automorphism will be given by a matrix of determinant 1 .

Proof. (1) follows immediately from Theorem 3 by taking $F_{i j}=\eta\left(E_{i j}\right)$ then, by the theorem we have a ring $U$ and a matrix $A \in U_{n}$ such that $A^{-1} B A=\eta(B), B \in C_{n}$.

(2) For a fixed $\eta$ we adjoin to $U$ the $n$th $r o o t$ of $a^{-1}=\operatorname{det}\left(A^{-1}\right)$ and replace $A$ by $a^{-1 / n} A$.

REMARK. The ring $U$ of Theorem 3 is not uniquely determined. For example one may take $U=\Pi C_{\mathrm{m}}$ where the product is taken over all maximal ideals in $C$. This ring will have the same property.

Corollary 5. Let $C$ be a commutative ring with unit, $\left\{F_{i j} \mid 1 \leqq\right.$ $i, j \leqq n\}$ a set of matrix units in $C_{n}$ then Cent $_{C_{n}}\left(F_{i j}\right)$-the centralizer of all the $F_{i j}$ in $C_{n}$ - is $C$, and every element in $C_{n}$ may be written in a unique way as $\sum_{i j} c_{i j} F_{i j}, c_{i j} \in C$. 
Proof. By Theorem $3 F_{i j}=A E_{i j} A^{-1}$ where $A$ is in some larger matrix ring $U_{n} \supseteqq C_{n}$. Hence; $\operatorname{Cent}_{C_{n}}\left(F_{i j}\right)=\operatorname{Cent}_{U_{n}}\left(F_{i j}\right) \cap C_{n}=$ $A \operatorname{Cent}_{U_{n}}\left(E_{i j}\right) A^{-1} \cap C_{n}=U \cap C_{n}=C$. The proof of the second part of the corollary is classical (e.g. [4], Proposition 6, p. 52).

3. The ring $V_{n}^{R}\left(R_{n}\right)$. Let $R$ be a fixed ring with unit. All rings henceforth will be $R$-algebras and all homomorphisms $R$ homomorphisms. We shall write $V_{n}(A)$ for $V_{n}^{R}(A)$. We now proceed to give an explicit construction for the ring $V_{n}\left(R_{n}\right)$.

Let $\left\{x_{i j} \mid 1 \leqq i, j \leqq n\right\}$ be $n^{2}$ commutative indeterminates over $R$, let $R\left[x_{i j}\right]$ denote the ring of polynominals in the $x_{i j}$ over $R$. Denote by $D \subseteq R\left[x_{i j}\right]$ the ideal generated in $R\left[x_{i j}\right]$ by the polynomial $\operatorname{det}\left(x_{i j}\right)-1$, and put $K=R\left[x_{i j}\right] / D$. Clearly we may take $R \cong K$. Put $\xi_{i j}=$ $x_{i j}+D \in K$, and $\Xi=\left(\xi_{i j}\right) \in K_{n}$. The matrix $\Xi$ is invertible in $K_{n}$, and its inverse is given by $\Xi^{-1}=\operatorname{adj} \Xi=\left(\hat{\Xi}_{j i}\right)$ where $\hat{\Xi}_{i j}$ is the algebraic complement of $\xi_{i j}$ in $\Xi$.

Let $S$ be the subalgebra of $K$ generated over $R$ by the $n^{4}$ elements $\left\{\xi_{i j} \hat{\Xi}_{k l} \mid 1 \leqq i, j, k, l \leqq n\right\}$. (S contains the unit element of $K$ for $\sum_{i=1}^{n} \xi_{1 i} \hat{\Xi}_{1 i}=\operatorname{det}\left(\xi_{i j}\right)=1$.)

Define a map $\rho: R_{n} \rightarrow K_{n}$ by $\rho(B)=\Xi B \Xi^{-1} . \quad \rho$ is clearly a unitary $R$ homomorphism, furthermore we have:

$$
\begin{aligned}
& {\left[\rho\left(E_{i j}\right)\right]_{k l}=\left(\Xi E_{i j} \Xi^{-1}\right)_{k l}=\sum_{\mu} \sum_{\nu}(\Xi)_{k \mu}\left(E_{i j}\right)_{\mu \nu}\left(\Xi^{-1}\right)_{\nu l} } \\
= & (\Xi)_{k i}\left(\Xi^{-1}\right)_{j l}=\xi_{k i} \hat{\Xi}_{l j} \in S
\end{aligned}
$$

so that $\rho\left(R_{n}\right) \subseteq S_{n}$ and we may regard $\rho$ as a map from $R_{n}$ to $S_{n}$ and note that the entries $\left\{[\rho(A)]_{i j} \mid A \in R_{n}\right\}$ generate $S$. For this ring $S$ and the homomorphism $\rho$ we prove;

THEOREM 6. $S$ is the universal ring $V_{n}\left(R_{n}\right)$ and $\rho$ is the canonical embedding of $R_{n}$ in $S_{n}=\left(V_{n}\left(R_{n}\right)\right)_{n}$.

Proof. We have seen that $S$ is generated by the appropriate elements so that all that remains to be shown is that every homomorphism $\tau: R_{n} \rightarrow C_{n}$ factors through $\rho$.

As $C$ is an $R$ algebra, we have the natural homomorphism $i: R \rightarrow C$. Denote $i(r)=r^{\prime} i_{n}(B)=B^{\prime}$ for all $r \in R, B \in R_{n}$.

Let $E_{i j}$ be the standard matrix units of $R_{n}$ than $E_{i j}^{\prime}$ are the standard matrix units of $C_{n}$ and $\tau\left(E_{i j}\right)=F_{i j}$ are a set of matrix units in $C_{n}$. By Theorem 3 there exists an $R$ algebra $U \supseteqq C$ and an invertible matrix $\left(a_{i j}\right)=A \in U_{n}$ such that $F_{i j}=A E_{i j}^{\prime} A^{-1}$; furthermore, by Corollary 4 (2) we may suppose that $\operatorname{det}(A)=1$. We clearly have for all $B \in R_{n} \tau(B)=A B^{\prime} A^{-1}$. 
Define $\eta^{\circ}: R\left[x_{i j}\right] \rightarrow U$ by $\eta^{\circ}\left(x_{i j}\right)=a_{i j}$, then $\eta^{\circ}\left(\operatorname{det}\left(x_{i j}\right)-1\right)=$ $\operatorname{det}\left(\eta^{\circ}\left(x_{i j}\right)\right)-1=\operatorname{det}(A)-1=0$, and therefore $\eta^{\circ}$ induces a homomorphism $\bar{\eta}: K=R\left[x_{i j}\right] / D \rightarrow U$ such that $\bar{\eta}\left(\xi_{i j}\right)=a_{i j}$, and we have the map $\bar{\eta}_{n}: K_{n} \rightarrow U_{n}$ for which $\bar{\eta}_{n}(\Xi)=A, \bar{\eta}_{n}\left(\Xi^{-1}\right)=A^{-1}$ and $\bar{\eta}_{n}(B)=$ $B^{\prime}$ for $B \in R_{n}$. For all $B \in R_{n}$ we have:

$\bar{\eta}_{n} \rho(B)=\bar{\eta}_{n}\left(\Xi B \Xi^{-1}\right)=\bar{\eta}_{n}(\Xi) \bar{\eta}_{n}(B) \bar{\eta}_{n}\left(\Xi^{-1}\right)=A B^{\prime} A^{-1}=\tau(B)$ so that $\bar{\eta}_{n} \rho=\tau$. Let $\eta$ be the restriction of $\bar{\eta}$ to $S$, then we have: $\rho\left(R_{n}\right) \subseteq$ $S_{n}$ and $\eta_{n}\left(S_{n}\right) \subseteq C_{n}$. The last inclusion follows from the fact that for $B \in R_{n} \quad \eta\left([\rho(B)]_{i j}=\left[\eta_{n} \rho(B)\right]_{i j}=[\tau(B)]_{i j} \in C\right.$ and since $S$ is generated by the elements $[\rho(B)]_{i j}, \eta(S) \subseteq C$. Consequently the following diagram is well defined and commutative.

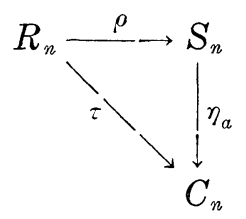

which completes the proof.

COROLlaRy 7. (1) If $R$ is an integral domain (in particular $R=F$ a field) then so is $V_{n}\left(R_{n}\right)$.

(2) If $R$ is noetherian so is $V_{n}\left(R_{n}\right)$.

Proof. (1) If $R$ is a domain then so is $R\left[x_{i j}\right]$ and the polynomial $\operatorname{det}\left(x_{i j}\right)-1$ is prime in $R\left[x_{i j}\right]$. Hence, $D$ is a prime ideal and $K=$ $R\left[x_{i j}\right] / D$ is a domain. $V_{n}\left(R_{n}\right)=S \subseteq K$ is hence also a domain.

(2) $S=V_{n}\left(R_{n}\right)$ is finitely generated over $R$ (see also [1]).

4. An alternative representation of $V_{n}\left(R_{n}\right), \quad V_{n}\left(R_{n}\right)$ was shown to be generated by $n^{4}$ elements $\xi_{k i} \hat{\Xi}_{l j}$. We aim now to describe the ring in terms of these generators and their relations. To this end we begin with a ring $R\left[z_{k l}^{i j}\right]$ with $z_{k l}^{i j} n^{4}$ commutative indeterminates over $R$. The elements $z_{k l}^{i j}$ are to represent the generators $\xi_{k i} \hat{\Xi}_{l j}$ and so they must satisfy the relations arising from the commutativity of the $\xi_{i j}$ and from the fact that $\Xi \Xi^{-1}=\Xi^{-1} \Xi=I$, namely;
(1) $z_{k l}^{i j} z_{t r}^{s q}=z_{k r}^{i q} z_{t l}^{s j}$
(2) $\sum_{i=1}^{n} z_{k l}^{i i}=\delta_{k l}$
(3) $\quad \sum_{k=1}^{n} z_{k k}^{i j}=\delta_{i j}$ for all $i, j, k, l, s, q, t, r$ for all $k, l$ for all $i, j$.

In fact we will show that the generators of $V_{n}\left(R_{n}\right)$ satisfy no other relations except these. This will be done by showing that $R\left[z_{k l}^{i j}\right]$ modulo those relations is again the universal ring $V_{n}\left(R_{n}\right)$.

We begin with conditions for matrices in a matrix ring to be matrix units. 
THEOREM 8. Let $\left\{F^{i j} \mid 1 \leqq i, j \leqq n\right\}$ be a set of $n^{2}$ matrices in a matrix ring $C_{n}$. The $\left\{F^{i j}\right\}$ are a set of matrix units in $C_{n}$ if and only if they satisfy the following conditions:

(1) $\left(F^{i j}\right)_{k l}\left(F^{s q}\right)_{t r}=\left(F^{i q}\right)_{k r}\left(F^{s j}\right)_{t l}$

(2) $\sum_{i=1}^{n}\left(F^{i i}\right)_{k l}=\delta_{k l}$

(3) $\quad \sum_{k=1}^{n}\left(F^{i j}\right)_{k k}=\delta_{i j}$

for all $i, j, k, l, s, q, t, r$ for all $k, l$ for all $i, j$.

Proof. Suppose the $F^{i j}$ are matrix units in $C_{n}$, then, by Theorem 3 we have $F^{i j}=A E_{i j} A^{-1}$ where $A \in U_{n}$, for some $U \supseteqq C$. Put $A=\left(a_{i j}\right)$ and $A^{-1}=\left(a_{i j}^{\prime}\right)$ and evaluate the left side of (1);

$$
\begin{aligned}
\left(F^{i j}\right)_{k l}\left(F^{s q}\right)_{t r} & =\left(A E_{i j} A^{-1}\right)_{k l}\left(A E_{s q} A^{-1}\right)_{t r} \\
& =\left(\sum_{\nu \mu} a_{k \nu}\left(E_{i j}\right)_{\nu \mu} a_{\ell l}^{\prime}\right)\left(\sum_{\pi \sigma} a_{t \pi}\left(E_{s q}\right)_{\pi \sigma} a_{\sigma r}^{\prime}\right) \\
& =\left(a_{k i} a_{j l}^{\prime}\right)\left(a_{t s} a_{q r}^{\prime}\right) .
\end{aligned}
$$

While the right side of (1) gives:

$$
\begin{aligned}
\left(F^{i q}\right)_{k r}\left(F^{s j}\right)_{t l} & =\left(A E_{i q} A^{-1}\right)_{k r}\left(A E_{s j} A^{-1}\right)_{t l} \\
& =\left(\sum_{\nu \mu} a_{k \nu}\left(E_{i q}\right)_{\nu \mu} a_{\mu r}^{\prime}\right)\left(\sum_{\pi \sigma} a_{t \pi}\left(E_{s j}\right)_{\pi \sigma} a_{\sigma l}^{\prime}\right) \\
& =\left(a_{k i} a_{q r}^{\prime}\right)\left(a_{t s} a_{j l}^{\prime}\right) .
\end{aligned}
$$

Which, by the commutativity in $U$ proves (1). To prove (2) we have only to notice that $\sum_{i=1}^{n}\left(F^{i i}\right)_{k l}=\left(\sum_{i=1}^{n} F^{i i}\right)_{k l}=(I)_{k l}=\delta_{k l}$. Condition (3) states that $\operatorname{tr}\left(F^{i j}\right)=\delta_{i j}$, now for $i \neq j$ we have

$$
\operatorname{tr}\left(F^{i j}\right)=\operatorname{tr}\left(F^{i i} F^{i j}\right)=\operatorname{tr}\left(F^{i j} F^{i i}\right)=\operatorname{tr}(0)=0=\delta_{i j}
$$

while for $i=j$ we have, using (1) and (2)

$$
\begin{aligned}
\operatorname{tr}\left(F^{i i}\right) & =1 \cdot \sum_{k=1}^{n}\left(F^{i i}\right)_{k k}=\left(\sum_{l=1}^{n}\left(F^{l l}\right)_{i i}\right)\left(\sum_{k=1}^{n}\left(F^{i i}\right)_{k l k}\right) \\
& =\sum_{l, k}\left(F^{l i}\right)_{i k}\left(F^{i l}\right)_{k i}=\sum_{l}\left(F^{l l}\right)_{i i}=1=\delta_{i i} .
\end{aligned}
$$

Conversely, suppose the $F^{i j}$ satisfy conditions $1,2,3$ than

$$
\begin{aligned}
{\left[\left(F^{\nu \mu}\right)\left(F^{\pi \sigma}\right)\right]_{k l} } & =\sum_{t=1}^{n}\left(F^{\nu \mu}\right)_{k t}\left(F^{\pi \sigma}\right)_{t l}=\sum_{t=1}^{n}\left(F^{\nu \sigma}\right)_{k l}\left(F^{\pi / t}\right)_{t t} \\
& =\left(F^{\nu \sigma}\right)_{k l} \sum_{t=1}^{n}\left(F^{\pi \mu}\right)_{t t}=\left(F^{\nu \sigma}\right)_{k l} \delta_{\pi \mu}=\left(\delta_{\pi \mu} F^{\nu \sigma}\right)_{k l} .
\end{aligned}
$$

This being true for all $k, l$ we have $F^{\nu \mu} F^{\pi \sigma}=\delta_{\pi \mu} F^{\nu \sigma}$. We also have $\left(\sum_{i=1}^{n} F^{i i}\right)_{k l}=\sum_{i=1}^{n}\left(F^{i i}\right)_{k l}=\delta_{k l}=(I)_{k l}$ hence $\sum_{i=1}^{n} F^{i i}=I$ which concludes the proof that the $F^{i j}$ are indeed matrix units. (A similar result on orthogonal idempotents was obtained in [7].)

We are finally in the position to present $V_{n}\left(R_{n}\right)$ in terms of 
generators and relations. We start as before with the ring $R\left[z_{k l}^{i j}\right]$ and let $J$ be the ideal generated by all the polynomials of the forms: $\boldsymbol{z}_{k l}^{i j} \boldsymbol{z}_{t r}^{s q}-\boldsymbol{z}_{k r}^{i q} \boldsymbol{z}_{t l}^{s j}, \sum_{i=1}^{n} \boldsymbol{z}_{k l}^{i i}-\delta_{k l}, \sum_{k=1}^{n} \boldsymbol{z}_{k k}^{i j}-\delta_{i j}$ where the indices range over all possible combinations. We denote by $\zeta_{k l}^{i j}$ the class $z_{k l}^{i j}+J$ in the quotient ring $S^{\prime}=R\left[x_{k l}^{i j}\right] / J=R\left[\zeta_{k l}^{i j}\right]$. We define now a homomorphism $\rho^{\prime}: R_{n} \rightarrow S_{n}^{\prime}$ by $\left[\rho^{\prime}(A)\right]_{k l}=\sum_{i=1}^{n} \sum_{j=1}^{n} a_{i j} \zeta_{k l}^{i j}$.

The relations imposed on the $\zeta_{k l}^{i j}$ and Theorem 8 imply that the matrices $\rho^{\prime}\left(E_{i j}\right)=\left(\zeta_{k l}^{i j}\right)$ are clearly a set of matrix units in $S^{\prime}$ so that evidently $\rho^{\prime}$ is indeed an $R$-homomorphism.

TheOREM 9. With these notations $S^{\prime}=V_{n}\left(R_{n}\right)$ and $\rho^{\prime}$ is the canonical embedding of $R_{n}$ in $S_{n}^{\prime}=\left(V_{n}\left(R_{n}\right)\right)_{n}$.

Proof. We note that $\left[\rho^{\prime}\left(E_{i j}\right)\right]_{k l}=\zeta_{k l}^{i j}$ so that $S^{\prime}$ is generated by the appropriate elements. All that remains to be shown is the universal property of $\left(S^{\prime} \rho^{\prime}\right)$. Let $\tau: R_{n} \rightarrow C_{n}$ be a homomophism, $F^{i j}=\tau\left(E_{i j}\right)$ are then a set of matrix units in $C_{n}$. Define $\tilde{\eta}: R\left[z_{k l}^{i j}\right] \rightarrow C_{n}$ by $\tilde{\eta}\left(z_{k l}^{i j}\right)=\left[\tau\left(E_{i j}\right)\right]_{k l}=\left(F^{i j}\right)_{k l}$, by Theorem $8 J \subseteq \operatorname{Ker} \tilde{\eta}$ and so $\tilde{\eta}$ induces a map $\eta: S^{\prime} \rightarrow C$ for which $\left.\eta_{(\zeta k l}^{(j i}\right)=\left[\tau\left(E_{i j}\right)\right]_{k l}$. It remains to show that $\eta_{n} \rho^{\prime}=\tau$, and clearly it is enough to demonstrate the equality on the generators $E_{i j}$ of $R_{n}$, indeed $\left[\eta_{n} \rho^{\prime}\left(E_{i j}\right)\right]_{k l}=\eta\left[\left[\rho^{\prime}\left(E_{i j}\right)\right]_{k l}\right]=\eta\left(\zeta_{k l}^{i j}\right)=$ $\left[\tau\left(E_{i j}\right)\right]_{k l}$. This being true for all $k, l$ and for all $i, j$ we have $\eta_{n} \rho^{\prime}=\tau$ as required.

REMARK. By the uniqueness of $V_{n}\left(R_{n}\right) S$ and $S^{\prime}$ should be isomorphic. The isomorphism is given by the correspondence $\theta: \zeta_{k l}^{i j} \rightarrow$ $\xi_{k i} \widehat{\Xi}_{l j}$ and $\rho=\theta \rho^{\prime}$.

5. Embedding in matrix rings of different order. In this section we investigate the homomorphisms of a matrix ring into matrix rings of higher orders. In particular we give a description of the ring $V_{m}\left(R_{n}\right)$ for all $n$ and $m$.

If $n$ and $m$ are integers such that $n / m$ we have an injection $\delta: C_{n} \rightarrow C_{m}$ which places an $n \times n$ matrix $m / n$-times along the diagonal of an $m \times m$ matrix. The combined map $R_{n} \stackrel{i_{n}}{\rightarrow} C_{n} \stackrel{\delta}{\rightarrow} C_{m}$ will be denoted by $\delta^{\prime}$.

Our first result is elementary:

Lemma 10. There exists a unitary $R$ homomorphism $\tau: R_{n} \rightarrow C_{m}$ if and only if $n / m$.

Proof. If $n / m$ we have exhibited such an homomorphism, namely $\delta^{\prime}$. Conversely, suppose there exists a $\tau: R_{n} \rightarrow C_{m}$. Let $\mathfrak{M}$ be a 
maximal ideal in $C$. We have the induced $R$ homomorphism $\bar{\tau}: R_{n} \stackrel{\tau}{\rightarrow}$ $C_{m} \stackrel{\pi_{m}}{\rightarrow}(C / \mathfrak{M})_{m}=K_{m}$, therefore, without loss of generality we may assume $C=K$ a field. Let $E_{i j}$ be the standard matrix units of $R_{n}$, then $\tau\left(E_{i j}\right)=f_{i j}$ are $n^{2}$ matrix units in $K_{m}$. We have $K_{n} \cong \sum_{i j} f_{i j} K \cong$ $K_{m}$ and $K_{m} \cong K_{n} \boldsymbol{\otimes}_{K} \operatorname{Cent}_{K_{m}}\left(K_{n}\right)$ by taking dimension over $k$ we clearly have $n / m$.

Consequently we can assert that $V_{m}\left(R_{n}\right) \neq\{0\}$ if and only if $m / n$, which we shall assume henceforth. We would like now to generalize Theorem 3 to the case of $n^{2}$ matrix units in a matrix ring of order $m$. Turning to subdirectly irreducible components does not seem to be very helpful and so we localize. Our next result is again not new. It was proved for example by Knus [5] in a more general setting. Our proof is rather elementary except for the use of the classical Skolem-Noether theorem.

Theorem 11. Let $(C, \mathfrak{M})$ be a local ring. $\left\{e_{i j}\right\}$ and $\left\{f_{i j}\right\}$ two sets of $n^{2}$ matrix units in $C_{m}(n / m)$, there exists an invertible matrix $A \in C_{m}$ such that $f_{i j}=A e_{i j} A^{-1}$ for $1 \leqq i, j \leqq n$.

Proof. $\left\{\pi_{m}\left(e_{i j}\right)\right\}$ and $\left\{\pi_{m}\left(f_{i j}\right)\right\}$ are two sets of $n^{2}$ matrix units in a matrix ring $(C / \mathfrak{M})_{m}$ over a field. By the Skolem-Noether theorem there exists an invertible matrix $\bar{y} \in(C / \mathfrak{M})_{m}$ such that $\pi_{m}\left(f_{i j}\right)=$ $\bar{y} \pi_{m}\left(e_{i j}\right) \bar{y}^{-1}$. Let $y$ be a matrix in $C_{m}$ with $\pi_{m}(y)=\bar{y} \cdot \quad 0 \neq \operatorname{det}\left(\pi_{m}(y)\right)=$ $\pi(\operatorname{det}(y)) \in C / \mathfrak{M}$, therefore, $\operatorname{det}(y) \notin \mathfrak{M}$ is invertible in $C$ and $y$ is an invertible matrix in $C_{m}$. Put $A=\sum_{\nu=1}^{n} f_{\nu 1} y e_{1 \nu}$ then we have

$$
1 \leqq i, j \leqq n \quad f_{i j} A=f_{i 1} y e_{1 j}=A e_{i j}
$$

furthermore:

$$
\begin{aligned}
\pi_{m}(A) \bar{y}^{-1} & =\sum_{\nu=1}^{n} \pi_{m}\left(f_{\nu 1}\right) \bar{y} \pi_{m}\left(e_{1 \nu}\right) \bar{y}^{-1}=\sum_{\nu=1}^{n} \pi_{m}\left(f_{\nu 1}\right) \pi_{m}\left(f_{\nu \nu}\right) \\
& =\sum_{\nu=1}^{n} \pi_{m}\left(f_{\nu \nu}\right)=\pi_{m}(I)=I,
\end{aligned}
$$

so $\pi_{m}(A)$ is invertible in $(C / \mathfrak{M})_{m}$ and hence, as above, $A$ is invertible in $C_{m}$ which, by (1), completes the proof of the theorem.

The next theorem can be proved with the help of Theorem 11 in the same way that we proved Theorem 3.

THEOREM 12. Let $C$ be a commutative ring with unit. $\left\{e_{i j}\right\}$ and $\left\{f_{i j}\right\}$ two sets of $n^{2}$ matrix units in $C_{m}(n / m)$. There exists a commutative ring $U \supseteqq C$ and an invertible matrix $A \in U_{m}$ such that $e_{i j}=A f_{i j} A^{-1}$ for all $1 \leqq i, j \leqq n$. The ring $U$ is independent of 
the $\left\{e_{i j}\right\}$ and $\left\{f_{i j}\right\}$. For fixed $\left\{e_{i j}\right\}$ we may choose the ring $U$ and the matrix $A$ in such a way that $\operatorname{det}(A)=1$.

We can now show that $V_{m}\left(R_{n}\right)$ is a subalgebra of $V_{m}\left(R_{m}\right)$, more precisely:

THeorem 13. Let $\rho: R_{m} \rightarrow\left(V_{m}^{R}\left(R_{m}\right)\right)_{m}$ and $\delta: R_{n} \rightarrow R_{m}$ be the canonical maps. Then $V_{m}^{R}\left(R_{n}\right)$ is the subalgebra $S$, generated in $V_{m}^{R}\left(R_{m}\right)$ by the entries $\left\{\left[\rho \delta(B)_{i j} \mid B \in R_{n}\right\}\right.$ and $\rho \delta$ is the corresponding canonical embedding.

Proof. The condition on the generators of $S$ is fulfilled by definition so we have only to show the factoring property for maps. Let $\tau: R_{n} \rightarrow C_{m}$ be a homomorphism, by the usual reasoning we have a ring $U \supseteqq C$ and a matrix $A \in U_{m}$ such that for $B \in R_{n} \quad \tau(B)=$ $A \delta^{\prime}(B) A^{-1}$ where $\delta^{\prime}$ is as in Lemma 10. Define $\tau^{\prime}: R_{m} \rightarrow U_{m}$ by $\tau^{\prime}(D)=A D^{\prime} A^{-1}$, we have the following commutative diagram:

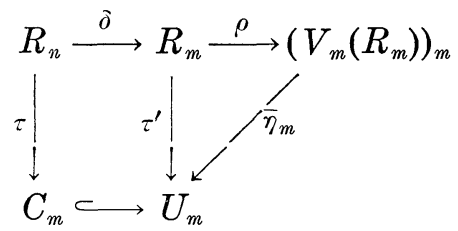

The square on the left is commutative by the definitions, while $\bar{\eta}$ and the commutativity of the triangle are given by the universality of $V_{m}\left(R_{m}\right)$.

Define $\eta$ to be the restriction of $\bar{\eta}$ to $S$. Then, for the generators of $S$ we have $\eta\left([\rho \delta(B)]_{i j}\right)=\left[\eta_{m} \rho \delta(B)\right]_{i j}=[\tau(B)]_{i j} \in C$. Therefore, $\eta(S) \subseteq C$ and the diagram

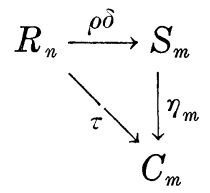

is well defined and commutative, which concludes the proof of the theorem.

REMARK. Obviously Corollary 7 may now be formulated for the ring $V_{m}^{R}\left(R_{n}\right)$. 


\section{REFERENCES}

1. S. A. Amitsur, Embedding in matrix rings, Pacific J. Math., 36 (1971), 21-29.

2. L. N. Childs and F. R. DeMeyer, On automorphisms of separable algebras, Pacific J. Math., 23 (1967), 25-34.

3. N. Divinsky, Commutative subdirectly irreducible rings, Proc. Amer. Math. Soc., 8 (1957), 642-648.

4. J. Jacobson, Structure of Rings, Amer. Math. Soc. Colloquium Publications, V. 23 1964.

5. M. A. Knus, Sur le théorème de Skolem-Noether et sur les dérivations des algébras d'Azumaya, C. R. Acad. Sc. Paris, 270 (1970), Série A, 637-639.

6. N. McCoy, Subdirectly irreducible commutative rings, Duke Math. J., 12 (1945), 381-387.

7. F. S. Nowlan, A note on primitive idempotent elements of a total matric algebra, Bull. Amer. Math. Soc., 37 (1931), 854-856.

Received July 5, 1972. This paper was written while the author was doing his Ph. D. thesis at the Hebrew University of Jerusalem, under the supervision of Professor

S. A. Amitsur, to whom he wishes to express his warm thanks.

The Hebrew University of Jerusalem 


\section{PACIFIC JOURNAL OF MATHEMATICS}

\section{EDITORS}

RICHARD ARENS (Managing Editor) University of California

Los Angeles, California 90024

R. A. Beaumon'T

University of Washington Seattle, Washington 98105
J. Dugundj1*

Department of Mathematics University of Southern California Los Angeles, California 90007

D. Gilbarg and J. Milgram Stanford University Stanford, California 94305

\section{ASSOCIATE EDITORS}

E. F. BECKENBACH

B. H. NeUMANN

F. WOLF

K. YOSHIDA

\section{SUPPORTING INSTITUTIONS}

UNIVERSITY OF BRITISH COLUMBIA CALIFORNIA INSTITUTE OF TECHNOLOGY UNIVERSITY OF CALIFORNIA MONTANA STATE UNIVERSITY UNIVERSITY OF NEVADA NEW MEXICO STATE UNIVERSITY OREGON STATE UNIVERSITY UNIVERSITY OF OREGON OSAKA UNIVERSITY

\section{UNIVERSITY OF SOUTHERN CALIFORNIA STANFORD UNIVERSITY UNIVERSITY OF TOKYO UNIVERSITY OF UTAH WASHINGTON STATE UNIVERSITY UNIVERSITY OF WASHINGTON AMERICAN MATHEMATICAL SOCIETY NAVAL WEAPONS CENTER}

* C. R. DePrima California Institute of Technology, Pasadena, CA 91109, will replace J. Dugundji until August 1974. 


\section{Pacific Journal of Mathematics}

\section{Vol. 49, No. $1 \quad$ May, 1973}

A. Bigard, Free lattice-ordered modules ...........................

Richard Bolstein and Warren R. Wogen, Subnormal operators in strictly cyclic

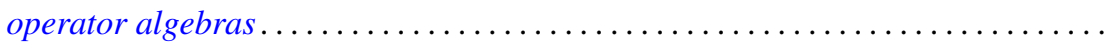

Herbert Busemann and Donald E. Glassco, II, Irreducible sums of simple

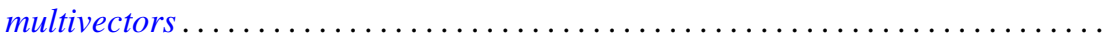

W. Wistar (William) Comfort and Victor Harold Saks, Countably compact groups

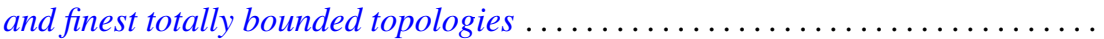

Mary Rodriguez Embry, Maximal invariant subspaces of strictly cyclic operator

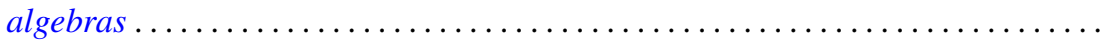

Ralph S. Freese and James Bryant Nation, Congruence lattices of semilattices......

Ervin Fried and George Grätzer, A nonassociative extension of the class of

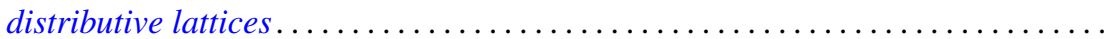

John R. Giles and Donald Otto Koehler, On numerical ranges of elements of locally

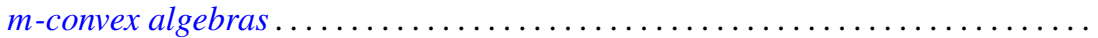

David A. Hill, On dominant and codominant dimension of $\mathrm{QF}-3$ rings ........ John Sollion Hsia and Robert Paul Johnson, Round and Pfister forms over $R(t) \ldots$ I. Martin (Irving) Isaacs, Equally partitioned groups . . . . . . . . . . . . . .

Athanassios G. Kartsatos and Edward Barry Saff, Hyperpolynomial approximation of solutions of nonlinear integro-differential equations.

Shin'ichi Kinoshita, On elementary ideals of $\theta$-curves in the 3-sphere and 2-links in the 4-sphere

Ronald Brian Kirk, Convergence of Baire measures

R. J. Knill, The Seifert and Van Kampen theorem via regular covering spaces ..

Amos A. Kovacs, Homomorphisms of matrix rings into matrix rings ..

Young K. Kwon, HD-minimal but no $H D$-minimal ..........

Makoto Maejima, On the renewal function when some of the mean renewal lifetimes are infinite

Juan José Martínez, Cohomological dimension of discrete modules over profinite groups.

W. K. Nicholson, Semiperfect rings with abelian group of units

Louis Jackson Ratliff, Jr., Three theorems on imbedded prime divisors of principal ideals.

Billy E. Rhoades and Albert Wilansky, Some commutants in $B(c)$ which are almost matrices

John Philip Riley Jr., Cross-sections of decompositions . . .

Keith Duncan Stroyan, A characterization of the Mackey uniformity $m\left(L^{\infty}, L^{1}\right)$ for

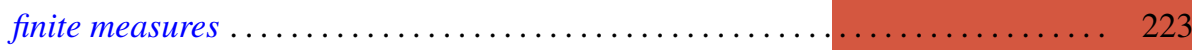

Edward G. Thurber, The Scholz-Brauer problem on addition chains . . . . . . . . . 229

Joze Vrabec, Submanifolds of acyclic 3-manifolds ............

Philip William Walker, Adjoint boundary value problems for compactified singular

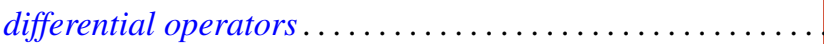

